Juvenal Soares Dias da Costa 1

Helen Gonçalves 2

Ana Maria B. Menezes 3

Eduardo Devens 4

Marcelo Piva 4

Maurício Gomes 4

Márcia Vaz 4

\section{Controle epidemiológico da tuberculose na cidade de Pelotas, Rio Grande do Sul, Brasil: adesão ao tratamento}

\author{
Epidemiological control of tuberculosis in Pelotas, \\ Rio Grande do Sul, Brazil: treatment compliance
}

1 Departamento de Medicina Social, Faculdade de Medicina, Universidade Federal de Pelotas. Av. Duque de Caxias 250, Pel otas, RS, 96030-002, Brasil.

2 Departamento de

Antropologia Social,

Universidade Federa

do Rio Grande do Sul.

Av. Bento Gonçalves 9500 ,

Porto Alegre, RS

90509-900, Brasil.

3 Departamento de Clínica

Médica, Faculdade de

Medicina, Universidade

Federal de Pelotas.

Av. Duque de Caxias 250,

Pel otas, RS, 96030-002, Brasil.

4 Faculdade de Medicina

Universidade Federal

de Pel otas.

Av. Duque de Caxias 250

Pel otas, RS, 96030-002, Brasil.
Abstract This study assesses risk factors for treatment noncompliance by patients regi stered with the Tuberculosis Control Program who live in the urban area of Pel otas, Rio Grande do Sul State. The study lasted from June 1994 to December 1995. All new cases diagnosed as pulmonary tuberculosis in the 20-80-year age bracket were monitored by the Tuberculosis Unit, hub of the tuberculosis control program in Pelotas. All patients was monitored from time of diagnosis through end of treatment, six months later. Patients answered a standard questionnaire. From June 1994 to June 1995, 152 cases were recorded, with some 20\% treatment noncompliance. We observed no significant association between noncompliance and employment status for headof-family, age, gender, al coholism, or presence of symptoms, while the only risk factor significantly associated with noncompliance was non-white skin color, probably due to the study's weak power as a function of sample size.

Key words Tuberculosis; Tuberculosis Control Program; Epidemiology

Resumo O objetivo deste estudo foi verificar fatores de risco para não-adesão ao tratamento para tuberculose nos pacientes inscritos no Programa de Controle da Tubercul ose, resi dentes na zona urbana da cidade de Pel otas. O estudo estendeu-se de jul ho de 1994 a dezembro de 1995. Uma coorte de paci entes com diagnóstico incidente de tuberculose, entre vinte e oitenta anos de idade, foi acompanhada no Centro de Saúde da cidade, onde são centralizados todos os pacientes com tuberculose. Cada paciente foi acompanhado desde o diagnóstico da doença atéo término do tratamento, seis meses após. Um questionário padronizado e pré-codificado foi aplicado a todos os pacientes. Foi diagnosticado um total de 152 casos novos de tuberculose no ano em estudo; destes, aproximadamente $20 \%$ dos mesmos abandonaram o tratamento. Em relação aos fatores de risco estudados para não-adesão ao tratamento não se encontrou signi fi cância estatística para fatores sóci o-econômicos, trabal ho do chefe da família, idade, sexo, al cooli smo e presença de sintomas. O único fator de risco que esteve estati sticamente associado com não-adesão ao tratamento foi cor não branca. Isto provavel mente ocorreu pela falta de poder do estudo em razão do tamanho da amostra.

Palavras-chave Tuberculose; Programa de Controle da Tuberculose; Epidemiologia 


\section{Introdução}

Segundo a Organização Mundial da Saúde (Crofton et al., 1992), em 1990, ocorreram oito milhões de novos casos de tuberculose no mundo, sendo 95\% em países em desenvolvimento e $5 \%$ nos países industrializados. O mais preocupante é que ocorrem aproximadamente três milhões de mortes, no mundo, por tuberculose em um ano. Estes números podem aumentar com a inter-relação da Aids e tuberculose. Antes da epidemia da Aids, nos países industrializados, a tuberculose era considerada como uma doença controlada e praticamente inexistente. É estimado, por exemplo, que, na próxima década, trezentos milhões de pessoas serão infectadas, noventa milhões desenvolverão a doença e trinta milhões morrerão por tuberculose (WHO, 1996).

No Brasil, anualmente, noventa mil doentes surgem e, destes, morrem cinco mil, número que não tem se alterado no decorrer dos anos. O País é o sexto com maior número de casos com tuberculose do mundo.

No Rio Grande do Sul, foram notificados 4.698 casos em 1994 (MS, 1995) e 4.893, em 1995 (SSMA, 1996). Em Pelotas, no período de julho de 1994 a julho de 1995, o setor de tisiologia do Centro de Saúde notificou cerca de 249 pacientes com tuberculose, abarcando a zona rural e cidades vizinhas de todas as idades. Segundo informantes da unidade local, Pelotas foi incluída entre os 250 municípios do País que deve intensificar o combate à doença por apresentar um alto índice de incidência da tuberculose.

O Programa de Controle da Tuberculose (PCT) preconiza como uma das estratégias de erradicação da doença interromper a cadeia de transmissão mediante a descoberta e o tratamento dos casos de tuberculose bacilíferos (Ott et al., 1993). Para se atingir este objetivo, torna-se essencial que se diagnostique o maior número possível de casos e que estes pacientes concluam o tratamento.

Um dos principais problemas encontrado pelo PCT refere-se à chamada adesão dos pacientes com tuberculose à terapêutica oferecida, ou seja, ao não concluírem o tratamento (por uma ou várias vezes), tornam-se pacientes crônicos, tanto da doença, quanto do serviço. A não-adesão ao tratamento é apontada como uma das graves falhas no programa para combater a doença.

A revisão da bibliografia a respeito da previsibilidade de adesão ao tratamento é controversa (Bayer \& Wilkinson, 1995). Autores americanos, como Curry (1964), já apontavam para menor adesão em pacientes de baixo nível sócio-econômico; outros, como Sbarbaro (1980), referiam que "infelizmente, o comportamento sani tário da mai oria das pessoas éimprevisível e não confirmam as nossas expectati vas de que sigam as recomendações médicas".

O objetivo deste estudo foi verificar a adesão ao tratamento para tuberculose e avaliar fatores de risco para não-adesão ao processo terapêutico, em pacientes residentes na zona urbana da cidade de Pelotas, no período de juIho de 1994 a dezembro de 1995.

\section{Metodologia}

O Projeto Controle Epidemiológico da Tuberculose na cidade de Pelotas - RS incluiu diversos subestudos. O presente subestudo teve como objetivo quantificar a não-aderência ao tratamento e identificar fatores de risco para a falta de adesão ao ele, por meio de uma análise quantitativa. Este trabalho está sendo complementado por estudo com método etnográfico, cujos resultados e análise não serão contemplados neste artigo.

Em Pelotas, todo paciente com suspeita de tuberculose é encaminhado ao Centro de Saúde da cidade e, havendo confirmação diagnóstica, o enfermo é inscrito no Programa de Controle da Tuberculose do Rio Grande do Sul (PCT/RS), vinculado à Secretaria da Saúde e do Meio Ambiente. A partir de normatização, a distribuição dos medicamentos tuberculostáticos são centralizados no Centro de Saúde da cidade. O tratamento é padronizado, durando, na maioria das vezes, seis meses (Palombini et al., 1996), e os pacientes recebem uma quantidade de medicação suficiente para um mês.

Desta forma, foi possível realizar um estudo de coorte com acompanhamento durante seis meses. De julho de 1994 a julho de 1995, todos os casos incidentes de tuberculose, de vinte a oitenta anos, residentes na zona urbana da cidade foram acompanhados no Centro de Saúde. Na primeira visita de cada paciente ao Centro de Saúde foi aplicado um questionário padronizado e pré-codificado contendo informantes relativas a variáveis sócio-econômicas, demográficas, hábitos de vida e apresentação da doença. Os questionários foram aplicados por estudantes de medicina submetidos previamente a programa de treinamento visando à padronização do método. Durante os seis meses subseqüentes, os pacientes retornavam mensalmente ao Centro de Saúde para a retirada dos medicamentos, sendo, então, verificada a adesão ao tratamento. Para o acompanha- 
mento da adesão, o estudo estendeu-se até dezembro de 1995.

As variáveis sócio-econômicas utilizadas foram: classe social (segundo a Associação Brasileira de Institutos de Pesquisa de Mercado, Abipeme), escolaridade e renda familiar. A classificação da Abipeme (Rutter, 1988) baseia-se na acumulação de bens materiais e na escolaridade.

Por fim, na análise bivariada, verificou-se que diversas células, principalmente referentes às categorias de base que significavam padrões sócio-econômicos mais elevados, ou maior renda e escolaridade, ficavam com valores inferiores a cinco, razão pela qual agruparam-se algumas categorias. Os indivíduos das classes sociais A, B e C, que totalizaram $16 \%$, foram agrupados para fins estatísticos. A variável escolaridade foi dicotomizada em alfabetizados e analfabetos. Da mesma forma, a renda familiar foi dividida em dois grupos: famílias recebendo até três salários mínimos e as com renda superior a três salários mínimos.

Além destas variáveis, coletaram-se informações sobre o trabalho dos pacientes e sua posição familiar (se era o chefe da família).

As variáveis demográficas estudadas foram sexo, idade e cor da pele (categorizada como brancos e não brancos).

Verificou-se o hábito de ingerir álcool por intermédio do questionário Michigan Al coholic Screening Test - MAST (Bailey et al., 1978). De acordo com o MAST os pacientes foram categorizados como escore alto ou escore baixo para alcoolismo.

Entre os sinais e sintomas pesquisados, levou-se em consideração a presença ou ausência de tosse, escarro, hemoptise, emagrecimento, anorexia, sudorese noturna e febre. Posteriormente, estes sinais ou sintomas foram agrupados na forma de somatório (Tabela 1). Presença de hemoptise foi analisada isoladamente por considerar-se um sinal representativo da gravidade da doença.

A variável dependente foi dicotomizada em pacientes curados (aqueles que completaram o tratamento e receberam alta por cura) e pacientes que não aderiram ou interromperam temporariamente o tratamento. O não-comparecimento à unidade de saúde para retirada dos medicamentos por mais de trinta dias foi categorizado como não-adesão.

Encontraram-se 152 casos e sete perdas, atingindo 4,6\% entre pacientes novos, tuberculosos entre vinte o oitenta anos de idade, acompanhados no período entre julho de 1994 a dezembro de 1995, residentes na zona urbana de Pelotas.
Tabela 1

Características da população do estudo em Pelotas, RS, 1994-1995.

\begin{tabular}{|c|c|c|}
\hline Variável & $\mathrm{n}$ & $\%$ \\
\hline \multicolumn{3}{|l|}{ Classe social } \\
\hline Classes $A+B+C$ & 25 & 16,4 \\
\hline Classe D & 47 & 30,9 \\
\hline Classe $\mathrm{E}$ & 80 & 52,6 \\
\hline \multicolumn{3}{|l|}{ Escolaridade } \\
\hline Alfabetizados & 132 & 86,8 \\
\hline Analfabetos & 20 & 13,2 \\
\hline \multicolumn{3}{|l|}{ Renda familiar } \\
\hline Mais de 3 salários mínimos & 56 & 36,8 \\
\hline A té 3 salários mínimos & 96 & 63,2 \\
\hline \multicolumn{3}{|l|}{ Trabalha } \\
\hline Sim & 60 & 39,5 \\
\hline Não & 92 & 60,5 \\
\hline \multicolumn{3}{|l|}{ Chefe da família } \\
\hline Sim & 86 & 56,6 \\
\hline Não & 66 & 43,4 \\
\hline \multicolumn{3}{|l|}{ Cor da pele } \\
\hline Branca & 101 & 66,4 \\
\hline Não branca & 51 & 33,6 \\
\hline \multicolumn{3}{|l|}{ Idade } \\
\hline De 20 a 29 anos & 37 & 24,3 \\
\hline De 30 a 39 anos & 51 & 33,6 \\
\hline De 40 a 49 anos & 33 & 21,7 \\
\hline De 50 ou mais anos & 31 & 20,4 \\
\hline \multicolumn{3}{|l|}{ Sexo } \\
\hline Feminino & 47 & 30,9 \\
\hline Masculino & 105 & 69,1 \\
\hline \multicolumn{3}{|l|}{ Alcoolismo (MAST) } \\
\hline Escore baixo & 119 & 78,3 \\
\hline Escore alto & 33 & 21,7 \\
\hline \multicolumn{3}{|l|}{ Número de sinais ou sintomas } \\
\hline Nenhum sinal ou sintoma & 6 & 3,9 \\
\hline 1 sinal ou sintoma & 2 & 1,3 \\
\hline 2 sinais ou sintomas & 5 & 3,3 \\
\hline 3 sinais ou sintomas & 15 & 9,9 \\
\hline 4 sinais ou sintomas & 24 & 15,8 \\
\hline 5 sinais ou sintomas & 33 & 21,7 \\
\hline 6 sinais ou sintomas & 52 & 34,2 \\
\hline 7 sinais ou sintomas & 15 & 9,9 \\
\hline \multicolumn{3}{|l|}{ Hemoptise } \\
\hline N ão & 110 & 72,4 \\
\hline Sim & 42 & 27,6 \\
\hline \multicolumn{3}{|l|}{ Tratamento completo } \\
\hline $\operatorname{Sim}$ & 122 & 80,1 \\
\hline Não & 30 & 19,9 \\
\hline
\end{tabular}


A entrada dos dados foi realizada por meio do Programa Epi-Info, duas vezes para se detectarem problemas de consistência. Procedeu-se análise univariada e bivariada no Programa SPSS. Utilizou-se como medida de efeito o risco relativo e verificou-se a precisão das medidas pelos intervalos de confiança a $95 \%$. O teste de diferenças entre proporções foi o Qui-Quadrado (Kirkwood, 1988).

O somatório dos sinais e sintomas foi analisado de acordo com a diferença de médias entre os pacientes que completaram e os que abandonaram o tratamento.

\section{Resultados}

Durante o estudo, trinta pacientes abandonaram o tratamento, dois $(6,7 \%)$ dos quais abandonaram no primeiro mês. Verificou-se que, em cada um dos três meses subseqüentes, oito $(26,7 \%)$ pacientes abandonaram o tratamento e nos últimos dois meses de tratamento observaram-se para cada um deles dois abandonos.

A Tabela 1 mostra que aproximadamente $20 \%$ dos pacientes inscritos no Programa de Tuberculose da cidade de Pelotas abandonaram o tratamento no período do estudo. Esta mesma tabela revela algumas características da população do estudo. Mais da metade dos casos pertenciam à classe $\mathrm{E}$ da Abipeme, ou ganhavam até três salários mínimos. Quanto ao grau de escolaridade, aproximadamente $87 \%$ dos indivíduos incluídos no estudo eram alfabetizados.

Conforme a Tabela 1, quanto às variáveis que investigavam aspectos de trabalho, constatou-se que $60 \%$ dos pacientes com a doença não exerciam atividades, verificando-se também que $60 \%$ ocupavam a posição de chefe na família.

Quanto à distribuição das variáveis demográficas, encontrou-se mais do que o dobro dos pacientes do sexo masculino. Em relação à idade, o maior contingente situou-se entre os trinta e 39 anos $(33,6 \%)$ e foi sucedido pelo grupo entre vinte e 29 anos (24,3\%). Verificou-se que $66 \%$ dos pacientes eram de cor branca.

A Tabela 1 ainda revela que mais de $20 \%$ dos casos atingiram um escore alto no questionário MAST para alcoolismo. Quanto à apresentação da doença, mais de $70 \%$ dos pacientes apresentavam quatro ou mais sinais ou sintomas de tuberculose, e cerca de $30 \%$ apresentaram hemoptise.

Na Tabela 2 estão apresentados os resultados da análise bivariada. Com exceção da cor não branca, que mostrou associação significa- tiva com não-adesão ao tratamento, as outras variáveis estudadas não mostraram associação significativa com não-adesão ao tratamento.

Observa-se, entretanto, um aumento dos riscos relativos em relação às respectivas categorias de base (risco relativo $=1,0$ ) nas variáveis classe social e escolaridade. Quanto pior a inserção de classe, maior o risco relativo para a não-adesão ao tratamento. Da mesma forma, observou-se um risco maior entre os casos não alfabetizados. Porém, esta tendência não foi constatada entre as pessoas que apresentavam renda familiar até três salários mínimos, entre os que trabal havam ou que não ocupavam a chefia nas famílias.

As variáveis demográficas revelaram que as pessoas acima de cinqüenta anos e os homens apresentavam um risco maior de não-adesão ao tratamento.

A variável que investigou cor da pele mostrou que as pessoas classificadas como não brancas apresentavam um risco relativo dobrado para não-adesão ao tratamento. Este achado foi confirmado pelos intervalos de confiança e mostrou diferença estatisticamente significativa.

Os casos com escore al to no questionário MAST e que apresentavam hemoptise tinham um maior risco de abandonar o tratamento.

Realizou-se análise de variância para estudar possíveis diferenças quanto à variável somatório dos sinais e sintomas. Não se constataram diferenças nas médias de sinais e sintomas entre os indivíduos que abandonaram e os que completaram o tratamento.

\section{Discussão}

Entre os casos inscritos no Programa de Controle da Tuberculose na cidade de Pelotas, encontraram-se $19,9 \%$ de pessoas que não aderiram ao tratamento. Para fins de comparação, encontrou-se na revisão bibliográfica uma taxa de $12,9 \%$ de não-adesão ao tratamento no Brasil, durante o período de 1981 e 1990 (Fiuza de Melo et al., 1993). Por sua vez, foram encontradas taxas de 11,9\% no Estado do Rio Grande do Sul, em 1988 (Gutierrez et al., 1993), e de 25\% na cidade de Porto Alegre, em 1995 (SMS, 1996).

Deve-se levar em consi deração que a efetividade do Programa de Controle da Tuberculose depende da capacidade de impedir a transmissão da doença, do percentual de pacientes que concluem tratamento e da eficácia dos medicamentos. Algumas publicações apontam que o impacto epidemiológico será atingido 
Fatores de risco para não-aderência ao tratamento para tuberculose. Pelotas, RS, 1994-1995.

\begin{tabular}{|c|c|c|c|c|}
\hline Variável & Não-adesão (\%) & R.R. & Intervalo de confiança & P-valor \\
\hline \multicolumn{5}{|l|}{ Classe social } \\
\hline Classes $A+B+C$ & $3(12,0)$ & 1,0 & & \\
\hline Classe D & $9(19,1)$ & 1,60 & $(0,47-5,37)$ & NS \\
\hline Classe E & $18(22,8)$ & 1,90 & $(0,61-5,92)$ & NS \\
\hline \multicolumn{5}{|l|}{ Escolaridade } \\
\hline Alfabetizados & $25(19,1)$ & 1,0 & & \\
\hline Analfabetos & $5(25,0)$ & 1,31 & $(0,57-3,03)$ & NS \\
\hline \multicolumn{5}{|l|}{ Renda familiar } \\
\hline Mais de 3 salários mínimos & $12(21,4)$ & 1,0 & & \\
\hline Até 3 salários mínimos & $18(18,9)$ & 0,88 & $(0,46-1,70)$ & NS \\
\hline \multicolumn{5}{|l|}{ Trabalha } \\
\hline Sim & $14(23,7)$ & 1,0 & & \\
\hline Não & $16(17,4)$ & 0,73 & $(0,39-1,39)$ & NS \\
\hline \multicolumn{5}{|l|}{ Chefe da família } \\
\hline Sim & $17(20,0)$ & 1,0 & & \\
\hline Não & $13(19,7)$ & 0,98 & $(0,52-1,88)$ & NS \\
\hline \multicolumn{5}{|l|}{ Corda pele } \\
\hline Branca & $15(14,9)$ & 1,0 & & \\
\hline Não branca & $15(30,0)$ & 2,02 & $(1,08-3,79)$ & $<0,05$ \\
\hline \multicolumn{5}{|l|}{ Idade } \\
\hline De 20 a 29 anos & $8(22,2)$ & 1,0 & & \\
\hline De 30 a 39 anos & $8(15,7)$ & 0,71 & $(0,29-1,71)$ & NS \\
\hline De 40 a 49 anos & $5(15,2)$ & 0,68 & $(0,25-1,88)$ & NS \\
\hline De 50 ou mais anos & $9(29,0)$ & 1,31 & $(0,57-2,97)$ & NS \\
\hline \multicolumn{5}{|l|}{ Sexo } \\
\hline Feminino & $7(15,2)$ & 1,0 & & \\
\hline Masculino & $23(21,9)$ & 1,44 & $(0,67-3,11)$ & NS \\
\hline \multicolumn{5}{|l|}{ Alcoolismo (MAST) } \\
\hline Escore baixo & $22(18,6)$ & 1,0 & & \\
\hline Escore alto & $8(24,2)$ & 1,30 & $(0,64-2,65)$ & NS \\
\hline \multicolumn{5}{|l|}{ Hemoptise } \\
\hline Não & $21(19,3)$ & 1,0 & & \\
\hline Sim & $9(21,4)$ & 1,11 & $(0,56-2,23)$ & NS \\
\hline
\end{tabular}

quando se alcançarem $70 \%$ de busca de casose a taxa de cura seja, no mínimo, de 85\% (Styblo, 1991) ou $90 \%$ (ACTE, 1993). Diante dos resultados deste estudo e destes parâmetros pode-se questionar a efetividade e a lógica que regem o Programa de Controle da Tuberculose na cidade de Pelotas. Os próprios parâmetros que avaliam os resultados do programa estabelecem que taxas de não-adesão acima de 15\% seriam consideradas inaceitáveis (Ott, 1993).

As estratégias de controle da Tuberculose têm sido elaboradas com o intuito de impedir a transmissão da doença em vez de procurar evitar o adoecimento (Penna, 1988). Assim, o Programa de Controle da Tuberculose foi desenhado seguindo um modelo centralizador, possível e necessário, em uma época de múltiplas instituições agindo na assistência à saúde (Ott et al., 1993). Inegavelmente este delineamento foi importante como estratégia de controle para a doença. A introdução de um tratamento de curta duração juntamente com o controle estabelecido pelo programa parece não abarcar outros fatores importantes que são de cunho social e, de modo geral, determinantes para um maior risco de adoecimento (Gui- 
Tabela 3

Tamanho de amostra necessário para demonstrar diferenças entre pacientes curados e que abandonaram o PCT, Pelotas, RS, $1994-1995$.

\begin{tabular}{|c|c|c|c|c|c|c|}
\hline Variável & Nível de confiança & Poder estatístico & $\begin{array}{l}\text { Razão expostos/ } \\
\text { não expostos }\end{array}$ & $\begin{array}{l}\text { Doentes não } \\
\text { expostos }\end{array}$ & Risco relativo & $\begin{array}{l}\text { Tamanho } \\
\text { da amostra }\end{array}$ \\
\hline Classe social & 0,95 & 0,8 & $2,0: 8,0$ & 0,12 & 2,0 & 575 \\
\hline Escolaridade & 0,95 & 0,8 & $8,5: 1,5$ & 0,25 & 2,0 & 360 \\
\hline Renda familiar & 0,95 & 0,8 & $4,0: 6,0$ & 0,21 & 2,0 & 178 \\
\hline Trabalha & 0,95 & 0,8 & $3,5: 8,5$ & 0,24 & 2,0 & 155 \\
\hline Cor da pele & 0,95 & 0,8 & $6,0: 4,0$ & 0,15 & 2,0 & 273 \\
\hline Idade & 0,95 & 0,8 & $2,0: 8,0$ & 0,22 & 2,0 & 254 \\
\hline Sexo & 0,95 & 0,8 & $6,0: 3,0$ & 0,22 & 2,0 & 174 \\
\hline Alcoolismo & 0,95 & 0,8 & $8,0: 2,0$ & 0,18 & 2,0 & 310 \\
\hline Hemoptise & 0,95 & 0,8 & $7,0: 3,0$ & 0,19 & 2,0 & 223 \\
\hline
\end{tabular}

marães, 1990), visto que a doença tem suas características agravadas em condições de vida mais fragilizadas. Na cidade de Pelotas, ainda hoje, segue-se um modelo vertical, centrado em um único local para cadastro, acompanhamento e controle do atendimento aos pacientes. Se aproximadamente trinta pacientes abandonaram o Programa no ano em estudo e não havia recursos disponíveis para seu rastreamento e reingresso, parece óbvio que, para efetivar o trabal ho, impõe-se a necessidade de nova estratégia de busca de faltosos e reingresso no tratamento. Na formulação desta nova estratégia, deve-se procurar mobilizar novos recursos dentro do sistema de saúde existente e da própria comunidade, ou seja, a descentralização do Programa, como aliás foi realizado com êxito em outros países em desenvolvimento (Wilkinson, 1994; Wilkinson \& Gilks, 1996). A própria distribuição quanto aos meses de abandono parece contribuir para essa nova estratégia. As manifestações clínicas tendem a diminuir nos primeiros quatro meses de tratamento, período no qual se concentrou mais de 80\% de não-adesão ao tratamento.

Em relação às características que poderiam prever os pacientes com risco de abandonar o programa, verificou-se que na grande maioria das variáveis os intervalos de confiança e os testes estatísticos não confirmaram as tendências apontadas pelos riscos relativos. Como o delineamento do estudo foi de coorte, pois um dos objetivos era medir a incidência notificada da doença (pacientes incluídos no programa em um ano), não se encontraram casos suficientes que pudessem constatar possíveis associações. A falta de poder para caracterizar diferenças nas variáveis estudadas está demonstrada na Tabela 3. No programa Epi-Info, cal- culou-se o tamanho de amostra segundo modelo para estudos de coorte para cada variável incluída na pesquisa. As estimativas de razão expostos:não expostos e prevalências da doença nos não expostos foram estabelecidas com base nos resultados da análise bivariada deste estudo. Verificou-se que todas as variáveis investigadas exigiam uma amostra de maior tamanho, o que provavelmente justifica a falta de significância estatística. Sendo assim, não foi possível identificar as características dos pacientes que não aderiram ao Programa de Tuberculose na cidade de Pelotas, durante o ano do estudo.

Independentemente desta falta de poder, publicaram-se os resultados na tentativa de apontar subsídios para futuros trabalhos.

De qualquer forma, deve-se ressaltar que as diferenças encontradas nas pessoas cuja cor da pele era não branca foram constatadas necessariamente com muita cautela, pois o diminuto tamanho de amostra impediu o controle de fatores de confusão. Certamente, em um modelo de análise hierarquizada para determinação de tuberculose, as variáveis sobredeterminantes seriam de natureza sócio-econômica. As características da amostra deste estudo confirmam que a tuberculose é uma doença associada à baixas condições de vida. No estudo de não-adesão do programa, deve-se também levar em consideração que as condições de vida da sociedade brasileira excluem as pessoas inseridas nas classes sociais mais baixas. Assim, os pacientes mais pobres, possivelmente não brancos, mais propensos a contraírem a doença, talvez sejam submetidos às condições mais adversas, dificultando uma adaptação pessoal e familiar ao período do tratamento. 


\section{Referências}

ACTE (Advisory Council for the Elimination of Tuberculosis), 1993. Initial Therapy for tuberculosis in the era of multidrug resistance: recommendations of the Advisory Council for the Elimination of Tuberculosis. Morbidity and Mortality Weekly Report, 2:RR/ 7.

BAILEY, W. C.; SELLERS, C. A.; SUTTON, F. D.; SHEEHY, T. W. \& MAETZ, M. H., 1978. Tuberculosis and alcoholism. A partial solution through detection. Chest, 73:183-185.

BAYER, R. \& WILKINSON, D., 1995. Directly observed therapy for tuberculosis: history of an idea. Lancet, 345:1.545-1.548.

CROFTON, J.; HORNE, N. \& MILLER, F., 1992. Clinical Tuberculosis. London: MacMillan Press Ltd.

CURRY, F., 1964. District clinics for out patient treatment of tuberculosis problem patients. British Journal Diseases of Chest, 46:524-530.

FIUZA DE MELLO, F. A.; IDE-NETO, J.; SEISCENTO, M.; PINTO, J. A. \& AFIUNE, J. B., 1993. Tuberculose multiresistente. Jornal de Pneumologia, 19:73-82.

GUIM ARÃES, R., 1990. Determinação social e doença endêmica. O caso da tuberculose. In: Textos de Apoio. Epidemiologia 1 (J. R. Carvalheiro, org.), pp. 211-233. Rio de Janeiro: Associação Brasileira de Saúde Coletiva (Abrasco)/Escola Nacional de Saúde Pública.

GUTIERREZ, R. S.; OTT, W. P. \& PICON, P. D., 1993. Prevenção e tratamento. In: Tuberculose: Epidemiologia, Diagnóstico eTratamento em Clínica eSaúde Pública (P. D. Picon, C. F. C. Rizzon \& W. P. Ott, orgs.), pp. 35-51. Rio de Janeiro: Ed. Médica e Científica Ltda.

KIRKWOOD, B., 1988. Essentials of Medical Statistics. Oxford: Blackwell Scientific Publications.

MS (Ministério da Saúde), 1995. Informe Epidemiológico do SUS. Brasília: Centro Nacional de Epidemiologia, Fundação Nacional de Saúde, MS

OTT, W. P.; KWITKO, M.; SOARES, I. P. \& GUIMARÃES, M.T., 1993. Programa de controle da tuberculose no Rio Grande do Sul. In: Tuberculose: Epidemiologia, Diagnóstico e Tratamento em Clínica e Saúde Pública (P. D. Picon, C. F. C. Rizzon \&W. P. Ott, orgs.), pp. 53-74. Rio de Janeiro: Ed. Médica e Científica Ltda.
OTT, W. P., 1993. Administração do programa. In:. Tuberculose: Epidemiologia, Diagnóstico e Tratamento em Clínica eSaúde Pública (P. D. Picon, C. F. C. Rizzon \& W. P. Ott, orgs.), pp. 117-125. Rio de Janeiro: Ed. Médica e Científica Ltda.

PALOMBINI, B. C.; HETZEL, J. L. \& CORREA DA SILVA, L. C., 1996. Tuberculose. In: Medicina Ambulatorial: Condutas Clínicas em Atenção Primária à Saúde (B. B. Ducan, M. I. Schmidt \& E. R. J. Giugliani, orgs.), pp. 352-358. Porto Alegre: Ed. Artes Médicas.

PENNA, M. L. F., 1988. Uma abordagem ecológica da tuberculose. Cadernos de Saúde Pública, 4:356362.

RUTTER, M., 1988. Pesquisa de Mercado. São Paulo: Ed. Ática.

SBARBARO, J. A., 1980. Public health aspects of tuberculosis: supervision of therapy. Clinics in Chest Medicine, 1:253-263.

SMS (Secretaria Municipal de Saúde), 1996. Boletim Epidemiológico, 1(3):2. Porto Alegre: Centro de Vigilância em Saúde.

SSM A (Secretaria da Saúde e do Meio Ambiente), 1996. Estatísticas de Saúde: Mortalidade, 1994. Porto Alegre: Núcleo de Informações em Saúde.

STYBLO, K., 1991. Epidemiology of Tuberculosis. Tuberculosis Association Sel ect Papers. Vol. 24, The Hague: Royal Netherlands.

WHO (World Health Organization), 1996. Tuberculosis. Fact Sheet N/ 104, March. Geneva: WHO.

WILKINSON, D., 1994. High-compliance tuberculosis treatment programme in a rural community. Lancet, 343:647-648.

WILKINSON, D. \& GILKS, C. F., 1996. Lessons to be learned from tuberculosis control in China. Lancet, 347:835. 